\title{
Deformation Behavior of Liquid Droplet in Shock-Wave-Induced Atomization
}

\author{
K. Mizuno ${ }^{1}$, T. Yada ${ }^{1}$, T. Kamiya ${ }^{1}$, M. Asahara*2, T. Miyasaka ${ }^{2}$ \\ ${ }^{1}$ Graduate School of Engineering, Gifu University, Gifu, Japan \\ ${ }^{2}$ Faculty of Engineering, Gifu University, Gifu, Japan \\ *Corresponding author email : asahara@gifu-u.ac.jp
}

\begin{abstract}
The objective of this study is to clarify the atomization behavior of a single droplet at high Weber number, which corresponds to catastrophic breakup. In this study, we compared the height and thickness of droplets in the initial stage of atomization by capturing single droplet atomization at high Weber number with high spatio-temporal resolution. The results show that the time variation of droplet height and thickness is independent of the Weber number. The wavelength of the droplet upstream interface from experiment roughly corresponds to that of the droplet upstream interface from the Rayleigh-Taylor instability theory.
\end{abstract}

\section{Keywords}

Deformation behavior, High Weber number, Droplet atomization, Shock wave, Catastrophic breakup

\section{Introduction}

The atomization morphology of a single droplet has been organized by Weber number and Ohnesorge number. For low-viscosity liquids such as water and hydrocarbon fuels (Ohnesorge number less than 0.1 ), the single droplet atomization is classified into five forms that are more dependent on Weber number than Ohnesorge number: vibrational breakup, bag breakup, multimode breakup, sheet-thinning breakup, and catastrophic breakup [1]. In catastrophic breakup, which is an atomization morphology at high Weber number, in addition to the atomization of the parent droplet surface, the parent droplet is broken up by the development of the corrugation at the upstream interface [2]. It is considered that the fragmentation of the parent droplet caused by the development of the corrugation generates a relatively large the liquid mass, which is then further atomized. By comparing experimental images with theory, Joseph et al. [3] indicated that the corrugation seen at the upstream interface arises from the Rayleigh-Taylor instability. However, the experimental images by Joseph et al. were unclear, and Theofanous et al. [4] argued that there is no corrugation at the droplet upstream interface and that it is a mirage caused by the shadowgraph method. The characteristics of the initial droplet deformation behavior in bag breakup, multimode breakup and shear breakup have been studied [5-7]. Wang et al. [8] investigated the droplet deformation characteristics at high resolution with high Weber number, and compared the results of the droplet deformation characteristics by changing the Mach number of the flow behind shock wave with constant Weber number. Therefore, the purpose of this paper is to investigate the characteristics of the initial deformation behavior of a single droplet at high Weber number corresponding to catastrophic breakup by filming the atomization experiment of a single droplet with even higher spatio-temporal resolution than that of Wang et al.

\section{Experimental Methods and Conditions}

Figure 1 shows the schematic of a droplet atomization observation system that uses a shock-wave tube. In this system, a single droplet falling at an arbitrary position is interfered with a shock wave; the droplet atomized in the high-speed airflow behind the shock wave is 
captured via a high-speed camera $(100,000 \mathrm{fps}, 1280 \times 448)$. The cross-section of the shock wave tube is a rectangle $(69 \mathrm{~mm} \times 69 \mathrm{~mm}$ ) with a width that allows the turbulent boundary layer behind the shock wave to not affect the atomization process. In order to accurately predict the position of the interference between the falling droplet and the shock wave, and to capture the image with a high-speed camera, we used the laser breaking method [9], which has excellent timing control of the shock wave generation. The system controls the timing of shock wave generation to within $\pm 0.1 \mathrm{~ms}$; thus, the system can capture the atomization behavior of a free-falling droplet with a positional accuracy of less than $0.1 \mathrm{~mm}$ in the vertical direction, which is sufficient for this study. A short-pulsed laser (Pulse width: $20 \mathrm{~ns}$, wavelength: $640 \mathrm{~nm}$ ) is used as the light source, used as a backlight through an optical fiber and a diffusion lens. Air is used as both the driving gas and the driven gas, and water is used as the liquid. The experimental conditions are shown in Table 1. In addition to the experiments at a high Weber number, experiments at a low Weber number corresponding to the sheet thinning breakup were also conducted for comparison.

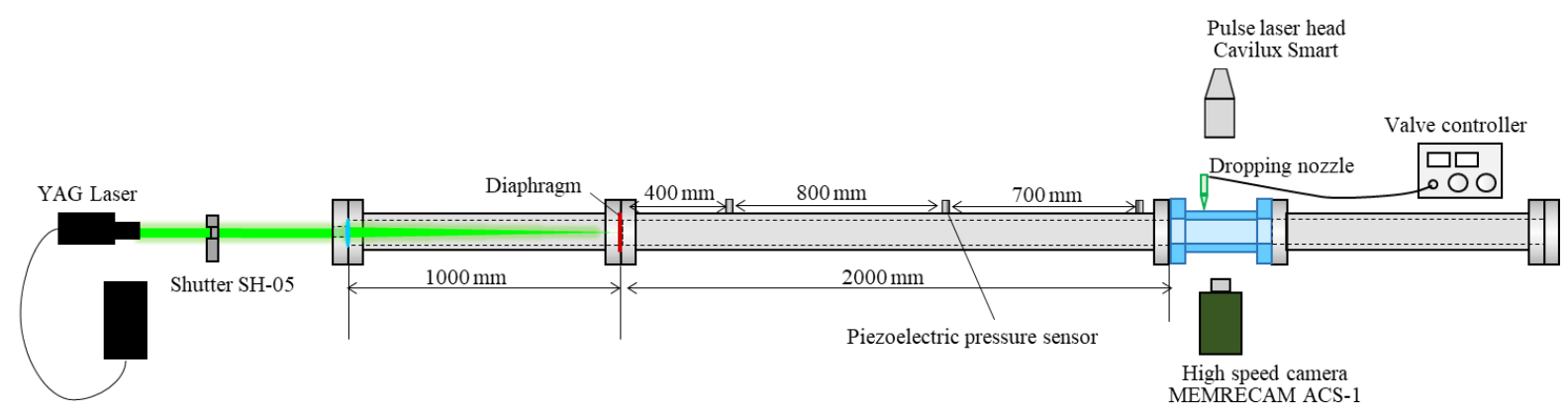

Figure 1. Schematic of the observation system of deformation and breakup of a liquid drop in high-speed gas flow using the shock tube operated by the laser rupture method.

Table 1 - Physical properties of single droplet atomization experiments using shock waves.

\begin{tabular}{|l|l|l|l|l|l|}
\hline Exp. \# & $\begin{array}{l}\text { Weber } \\
\text { number, } W e \\
{[-]}\end{array}$ & $\begin{array}{l}\text { velocity behind } \\
\text { shock wave, } \\
u_{2}[\mathrm{~m} / \mathrm{s}]\end{array}$ & $\begin{array}{l}\text { Mach number } \\
\text { behind shock } \\
\text { wave, } M_{2}[-]\end{array}$ & $\begin{array}{l}\text { Density behind } \\
\text { shock wave, } \rho_{2} \\
{\left[\mathrm{~kg} / \mathrm{m}^{3}\right]}\end{array}$ & $\begin{array}{l}\text { Temperature } \\
\text { behind shock } \\
\text { wave, } T_{2}[\mathrm{~K}]\end{array}$ \\
\hline 1 & 1316 & 149.5 & 0.40 & 1.83 & 345.7 \\
\hline 2 & 2010 & 178.2 & 0.47 & 1.96 & 358.7 \\
\hline 3 & 2680 & 201.1 & 0.52 & 2.07 & 368.6 \\
\hline 4 & 214 & 67.2 & 0.19 & 1.47 & 314.4 \\
\hline
\end{tabular}

\section{Results and Discussion}

Figure 2 illustrates the atomization behavior of a single droplet taken with the experimental equipment (Figure 1). The time when the shock wave reaches the droplet is defined as $t=0$ $\mathrm{ms}$, and the time required for a rigid sphere to move its own diameter in the airflow [10],

$$
t_{d}=\sqrt{\frac{8 \rho_{l}}{3 C_{D} \rho_{g}}} \frac{d_{0}}{u_{g}}
$$

which is non-dimensionalized time as $t / t_{\mathrm{d}}$, is also shown. The height $Y$ and thickness $X$ of the parent droplet and fragments originating from the parent droplet shown in Figure 3 are nondimensionalized by the initial droplet diameter. Figure 4 depicts the graph of non-dimensional time $t / t_{\mathrm{d}}$. Trend are similar up to $t / t_{d}=0.15$ for a high Weber number in Figure $4(\mathrm{a})$. After $t / t_{\mathrm{d}}=$ 
$0.15, Y / d_{0}$ decreases at $W e=1316$ and $W e=2010$. This is because the fragments are stripped from the top and bottom of the parent droplet and flow downstream. Further, height $Y / d_{0}$ is smaller at low Weber numbers than that at high Weber numbers. As shown in Figure 4(b), there is no significant difference based on the Weber number in the range of this Weber number condition. However, for mass conservation, if there is no significant difference in the thickness $X / d_{0}$ in the horizontal direction to the flow, there should be no significant difference in the elongation $Y / d_{0}$ in the vertical direction to the flow. Figure 2 shows that the fine particles are stripped from the parent droplet at $t / t_{d}=0.11$ at a high Weber number; the stripped fine particles cover the parent droplet, which makes it impossible to capture the parent droplet correctly, thereby resulting in the overestimation of $Y / d_{0}$.

The wavelength of the corrugations upstream of the parent droplet interface is compared to the wavelength from the Rayleigh-Taylor instability theory. The growth factor of the RayleighTaylor instability [2] is given by,

$$
n=-k^{2} \frac{\mu_{2}+\mu_{1}}{\rho_{2}+\rho_{1}} \pm\left[k \frac{\rho_{2}-\rho_{1}}{\rho_{2}+\rho_{1}} a-\frac{k^{3} \sigma}{\rho_{2}+\rho_{1}}+k^{4}\left(\frac{\mu_{2}+\mu_{1}}{\rho_{2}+\rho_{1}}\right)^{2}\right]^{1 / 2},
$$

where $\rho_{1}$ and $\rho_{2}$ represent the density of the gas and the density of the liquid, respectively; $\mu_{1}$ and $\mu_{2}$ denote the viscosity coefficients of the gas and liquid, respectively; $k$ denotes the wave number of the disturbance; $\sigma$ represents the surface tension; and $a$ denotes the acceleration. In this study, because air is used as a gas and water as a liquid, $\mu_{1} \approx 0, \mu_{2} \approx 0$, and $\rho_{1} \approx 0$. Therefore,

$$
n=\left[k a-\frac{k^{3} \sigma}{\rho_{2}}\right]^{1 / 2}
$$

was used as the growth factor. From this equation, the wavelength at which the growth factor is maximized is

$$
\lambda^{*}=2 \pi \sqrt{\frac{3 \sigma}{\rho_{2} a}} .
$$

The acceleration $a$ is obtained by fitting $x=1 / 2 a t^{2}$ from the $x-t$ diagram, where $x$ denotes the movement of the upstream of the parent droplet interface at a certain time $t$. Table 2 lists the accelerations obtained from the fitting. The plot points shown in Figure $\mathbf{5}$ are obtained from the experimental images, and the solid lines are obtained from the fitting equations. Using this acceleration $a$, we obtain the wavelength $\lambda^{*}$ at which the growth factor is the maximum based on Eq. (3). Figure 6 illustrates the comparison between the wavelength of the corrugation at the upstream interface obtained from the experimental images and wavelength $\lambda^{*}$ obtained from the Rayleigh-Taylor instability theory at each Weber number. As reported in Joseph et al., the wavelength of the corrugations at the upstream interface of the droplet obtained experimentally and theoretically agree with each other (Table 3 ). 
(a) $W e=1316$

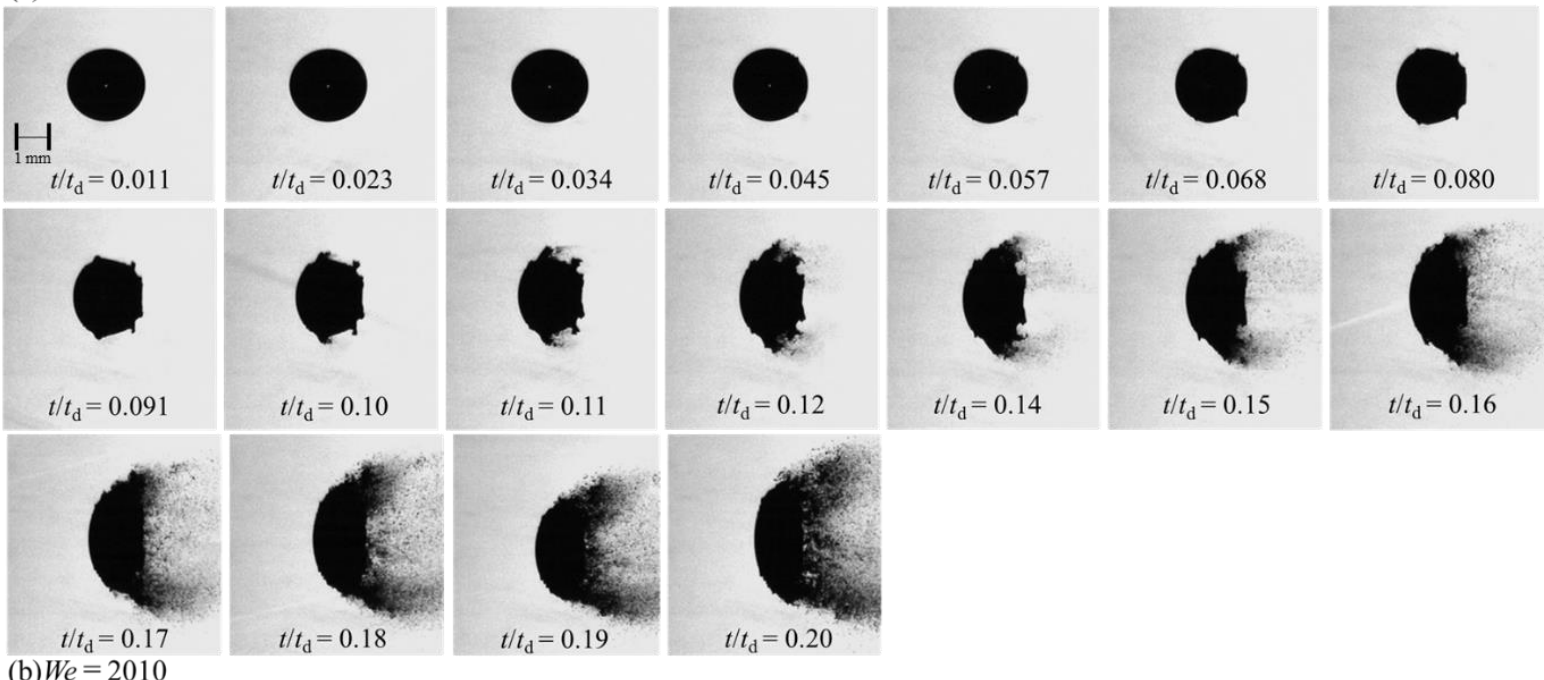

(b) $W e=2010$
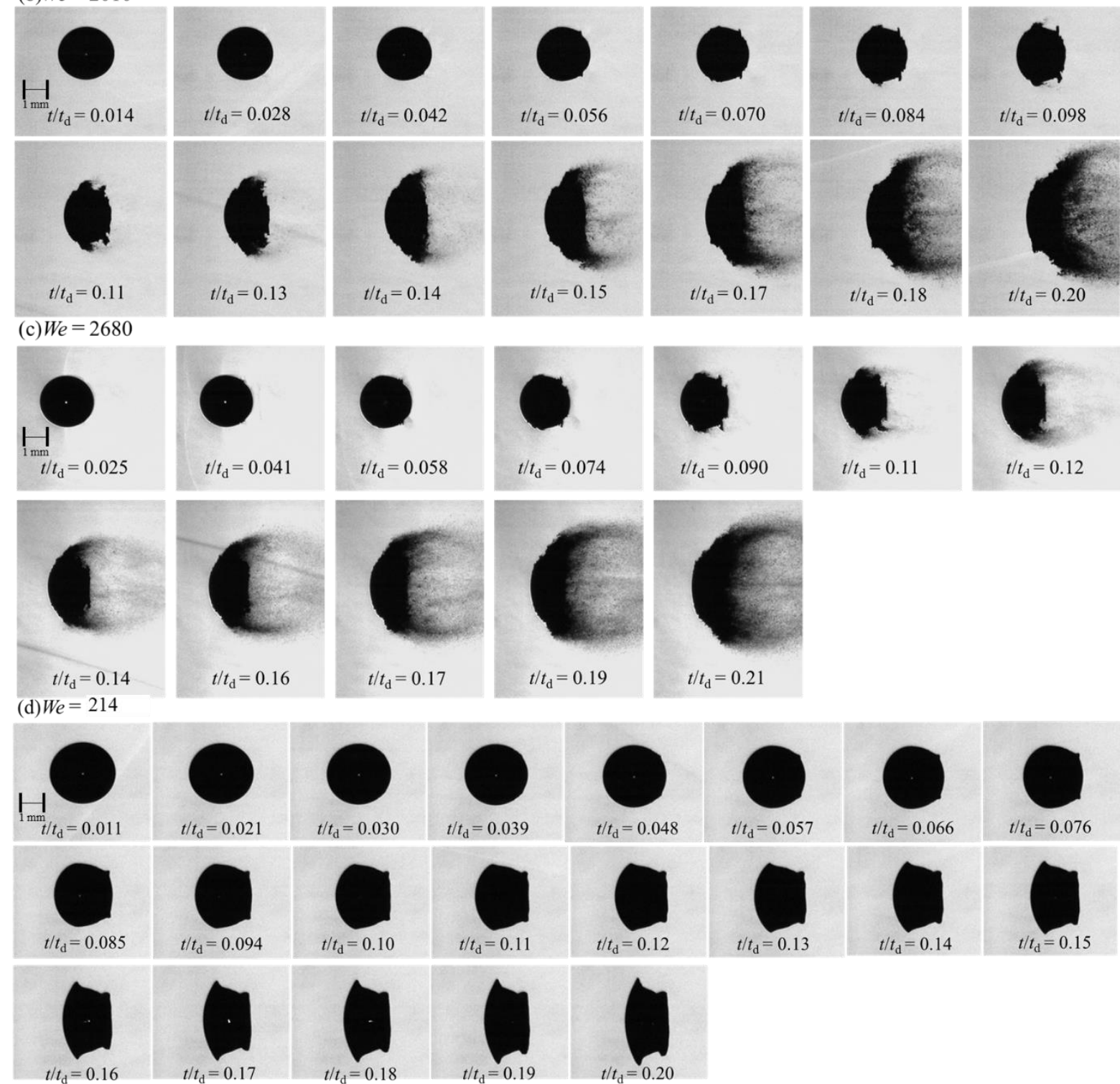

Figure 2. Atomization behavior of a single droplet by flow behind the shock wave at each Weber number. For the sheet-thinning breakup, fine droplets are not observed even at the dimensionless time when fine droplets are observed in the catastrophic breakup. (a): $W e=1316$ in catastrophic breakup, (b): $W e=2010$ in catastrophic breakup, (c) $W e=2068$ in catastrophic breakup, and (d) We $=214$ in sheet-thinning breakup. 


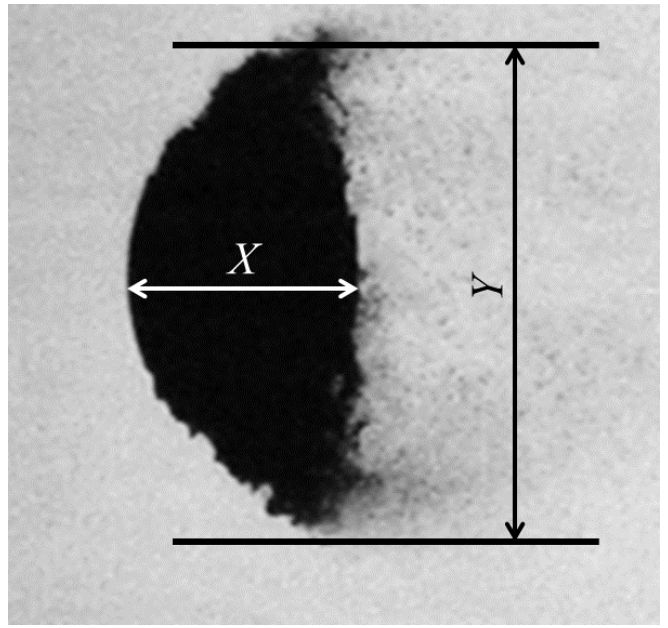

Figure 3. Definition of height $Y$ and thickness $X$ of the parent droplet at a given time in the droplet atomization process.

(a)

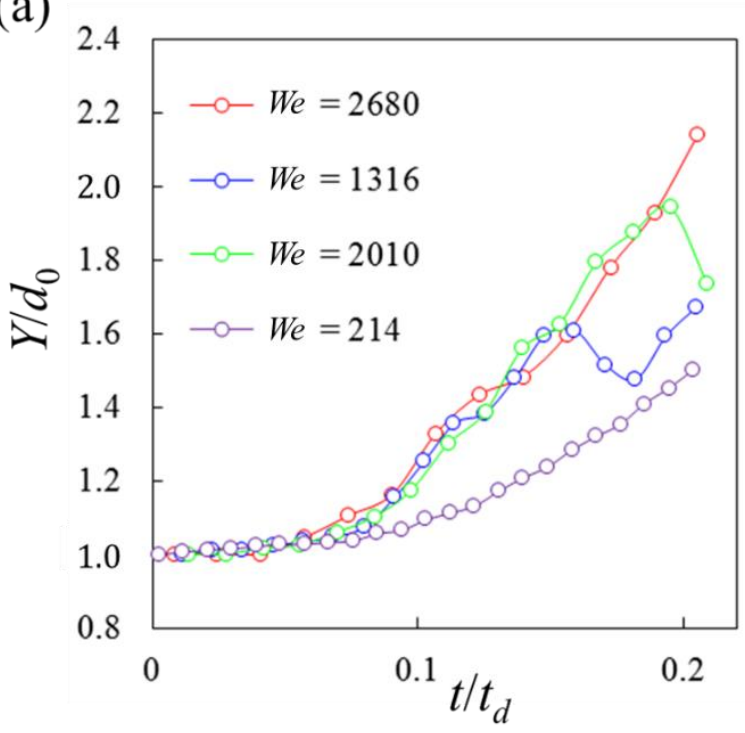

(b)

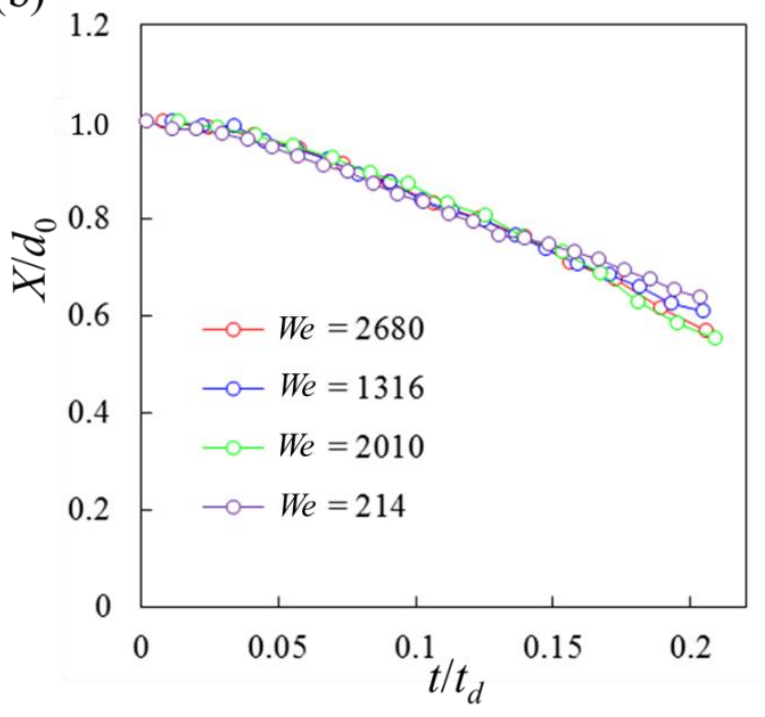

Figure 4. Plot of $Y / d_{0}$ and $X / d_{0}$ versus dimensionless time $t / t_{\mathrm{d}}$, where height $Y$ and thickness $X$ are nondimensionalized by the initial droplet diameter.
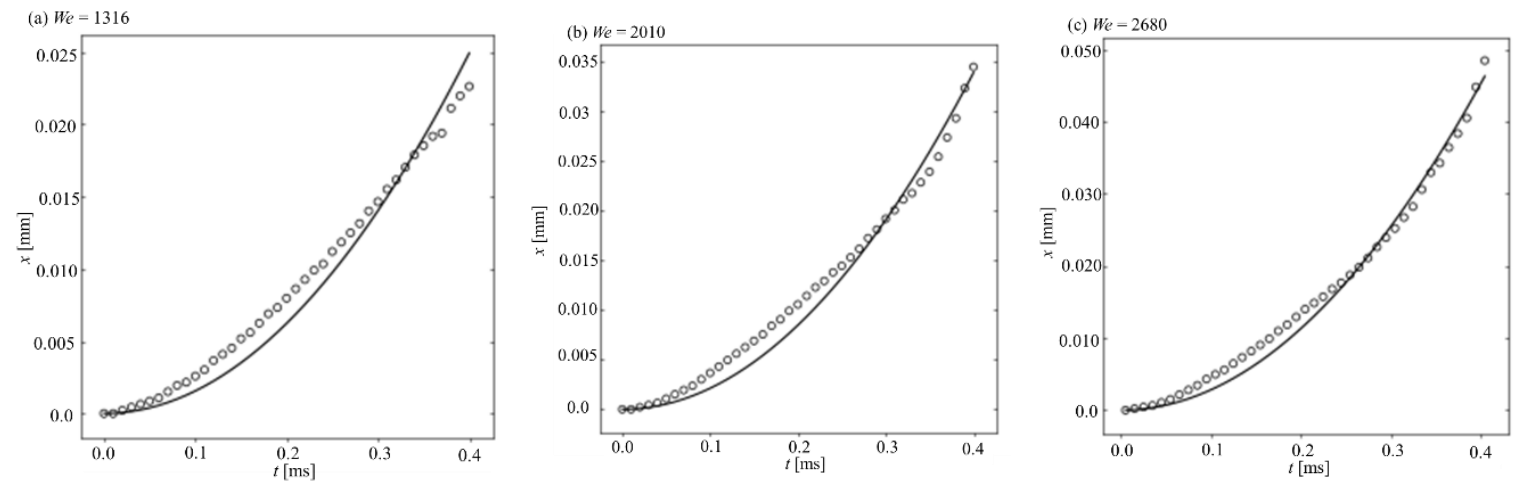

Figure 5. Graph of droplet movement at the upstream interface $x$ vs. time $t$ : The plotted points show the results obtained from the experimental images, and the solid line shows the result of fitting $x=1 / 2 a t^{2}$. 
Table 2 - Acceleration $a\left[\mathrm{~m} / \mathrm{s}^{2}\right]$ of the droplet obtained by fitting the equation $x=1 / 2 a t^{2}$ at each Weber number.

\begin{tabular}{|l|l|l|l|}
\hline Weber number & $W e=1316$ & $W e=2010$ & $W e=2680$ \\
\hline acceleration $a\left[\mathrm{~m} / \mathrm{s}^{2}\right]$ & $7.8 \times 10^{3}$ & $1.1 \times 10^{4}$ & $1.4 \times 10^{4}$ \\
\hline
\end{tabular}

(a) $W e=1316$

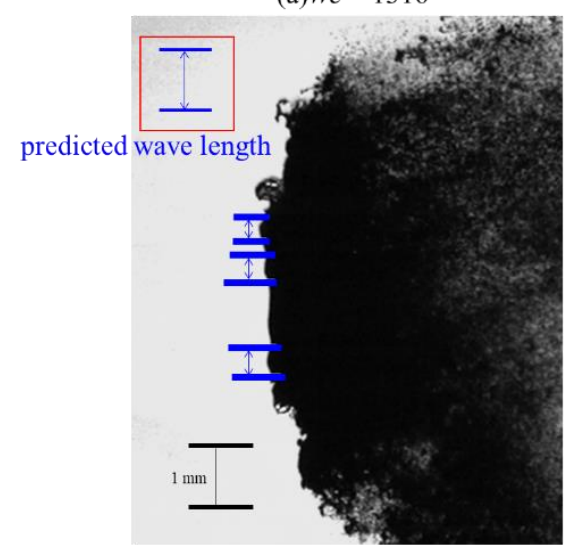

(b) $W e=2010$

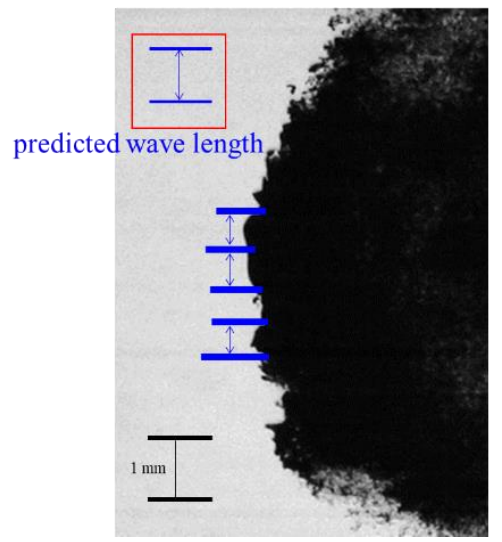

(c) $W e=2680$

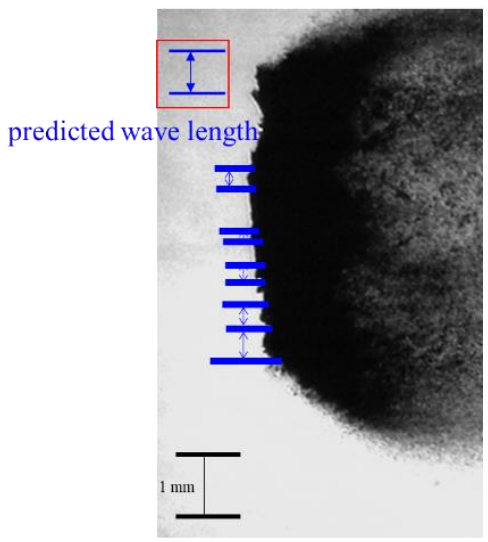

Figure 6. Wavelengths of the upstream interface corrugations obtained from the experimental result and theoretical wavelengths of the Rayleigh-Taylor instability. The tick marks on the photographs locate wave troughs.

Table 3 - Results for the wavelength of the disturbance obtained from the experimental results and the theoretical wavelength of the Rayleigh-Taylor instability at each Weber number.

\begin{tabular}{|c|c|c|c|c|c|c|}
\hline & $\begin{array}{l}\text { Theoretical } \\
\text { wavelength, } \\
\lambda^{*}[\mathrm{~mm}]\end{array}$ & $\begin{array}{l}\text { wave1, } \\
\lambda_{1}[\mathrm{~mm}]\end{array}$ & $\begin{array}{l}\text { wave2, } \\
\lambda_{2}[\mathrm{~mm}]\end{array}$ & $\begin{array}{l}\text { wave3, } \\
\lambda_{3}[\mathrm{~mm}]\end{array}$ & $\begin{array}{l}\text { wave4, } \\
\lambda_{4}[\mathrm{~mm}]\end{array}$ & $\begin{array}{c}\text { wave5, } \\
\lambda_{5}[\mathrm{~mm}]\end{array}$ \\
\hline$W e=1316$ & 1.05 & 0.45 & 0.39 & 0.31 & - & - \\
\hline$W e=2010$ & 0.90 & 0.57 & 0.62 & 0.50 & - & - \\
\hline$W e=2680$ & 0.78 & 0.27 & 0.19 & 0.20 & 0.34 & 0.50 \\
\hline
\end{tabular}

\section{Conclusions}

In this study, we focus on the catastrophic breakup, which is a form of atomization at high Weber number, and measure the height $Y / d_{0}$ and thickness $X / d_{0}$ at the initial deformation of the droplet. The results show that the same tendency is observed for the height at high Weber number, which is larger than that at low Weber number, indicating that the fine particles from the parent droplet cause the height to be estimated larger at high Weber number. In terms of thickness, no difference was observed between the Weber numbers under the conditions of this experiment. Additionally, the wavelengths of the corrugations observed at the upstream interface measured from the experimental images were compared with those obtained from the theory of the Rayleigh-Taylor instability, and some agreement was obtained.

\section{Acknowledgments}

This work was supported by JSPS KAKENHI Grant Number JP19J13109 and Grant-in-Aid for Research on Atomization and Spray Systems from ILASS-Japan, No. 2020-02.

\section{Nomenclature}

$\begin{array}{ll}C_{\mathrm{D}} & \text { drag coefficient [ - ] } \\ W e & \text { Weber number [ - ] } \\ X & \text { thickness of parent droplet [m] } \\ Y & \text { height of parent droplet [m] } \\ a & \left.\text { acceleration [m s }{ }^{-2}\right]\end{array}$




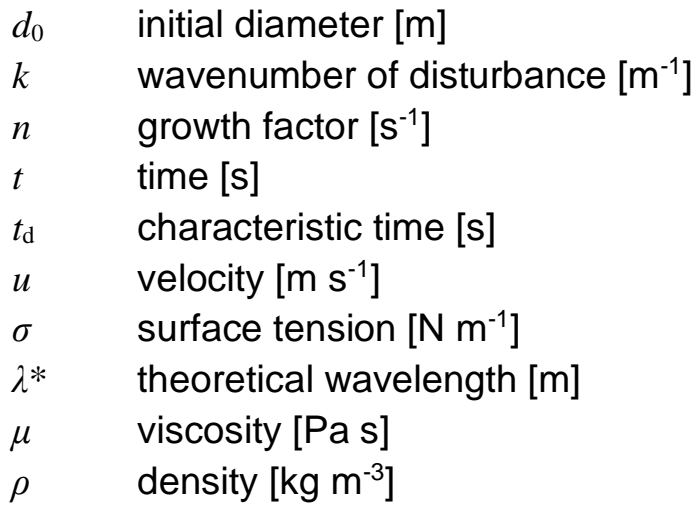

\section{References}

[1] Guildenbecher, D.R., Lopez-Rivera, C., Sojka, P.E., 2009, Experiments in Fluids, 46, pp. 371-402.

[2] Lee, C.H., Reits, R.D., 1999, International Journal of Multiphase Flow, 26, pp.229-244.

[3] Joseph, D.D., Belanger, J., Beavers, G.S., 1999, International Journal of Multiphase Flow, 25, pp.1263-1303.

[4] Theofanous, T.G., Li, G.J., 2008, Physics of Fluids, 20, 052103.

[5] Chou, W.H., Hsiang, L.P., Faeth, G.M., 1997, International Journal of Multiphase Flow, 23, pp.651-669.

[6] Chou, W.H., Faeth, G.M., 1998, International Journal of Multiphase Flow, 24, pp.889-912.

[7] Dai, Z., Faeth, G.M., 2001, International Journal of Multiphase Flow, 27, pp.217-236.

[8] Wang, Z., Hopfes, T., Giglmaier, M., Adams, N.A., 2020, Experiments in Fluids, 61(193), pp.1-17.

[9] Asahara, M., Hattori, Y., Miyasaka, T., Kamiya, T., 2020, Transactions of the JSME (in Japanese), 86,pp.1-12

[10] Kamiya, T., Asahara, M., Hattori, Y., Miyasaka, T., Asato, K., 2019, Atomization (in Japanese), 28, pp.32-39. 